\title{
Gait Recognition Based on Silhouette, Contour and Classifier Ensembles
}

\author{
M. Romero-Moreno, J. Fco. Martínez-Trinidad, and J.A. Carrasco-Ochoa \\ Computer Science Department, INAOE, Apdo. Postal $51 \& 216$ \\ Tonantzintla, Puebla, México \\ \{rmiriam, fmartine, ariel\}@inaoep.mx
}

\begin{abstract}
Gait Recognition is a non-invasive biometric technique for identifying persons through the way they walk. Currently there are many Gait Recognition methods, most of them based on a similarity function. In this paper, we propose two new methods for Gait Recognition based on silhouette and contour, using a classifier ensemble. Experimental results on a public standard database are shown and compared against others Gait Recognition methods.
\end{abstract}

Keywords: Computer vision, Gait recognition, Classifier ensembles.

\section{Introduction}

Biometrics refers to the automatic recognition of an individual by using anatomical or behavioral traits associated with that person [1]. Gait recognition (GR) is a biometric technique for recognizing persons through the particular way they walk [2,3]. Some of the advantages of GR are that it is a non-invasive technique, it is difficult to conceal, it can be done at a distance, and it does not need high resolution images.

There are complementary studies that support the idea of GR, such as medical studies focused on therapies, biomechanics, including studies in psychology supporting the idea that persons can be recognized by the way they walk [4]. Clearly, GR has some limitations as a biometric method, because shoes and clothing could affect the gait, as well as some physical conditions such as pregnancy, pain, disease, etc. These factors are well known and the majority of the biometrical methods have to deal with them. For example, in face recognition, a person may wear glasses or a moustache; may have a plastic surgery, etc. GR could be applied in controlled environments as banks, military installations, hotels, etc. In general, places where a fast detection of threats is required [5].

In GR, images from a video sequence are processed for obtaining data that reflect the identity of the person who is walking. There are two main approaches for GR: model-based and motion-based. Model-based GR methods use information gathered from the human body to construct a model for recognition. In general, model-based GR methods are not robust to position and scale changes and require high quality gait sequences. Thus, some model-based GR systems require multiple cameras for collecting body information. In motion-based GR methods, the motion information is directly used. Motion-based methods commonly use sequences of binary silhouettes 
extracted from the video sequence, using segmentation techniques such as background subtraction [6].

In this paper, we are interested in those methods that resolve the GR problem using sequences of binary silhouettes, because those methods do not require details such as texture or color, and they are not too much sensible to illumination.

This paper is organized as follows; in section 2 some recent GR methods are reviewed. Section 3 describes the proposed methods for Gait Recognition. The experimental results are presented in section 4 and finally the conclusions and future work are discussed in section 5 .

\section{Related Work}

In this section, some recent GR methods are reviewed, showing the most relevant details of each method.

Wang, Tan, Ning and $\mathrm{Hu}$ (2003) [7], introduced a GR method based on silhouette extraction. The silhouettes are extracted using background subtraction, and then the outer contour and shape centroid are obtained. After, a vector with all the distances between each contour pixel and the centroid is built. Then, PCA is used for reducing dimensionality. Recognition is done by measuring the distances between the training sequences and the sequence to be classified and applying the nearest neighbor classifier.

Lui and Sarkar (2004) [8], proposed a GR method also based on extracting the silhouettes and dividing the sequence in steps using the gait period length. For calculating the gait period length, the number of silhouette pixels of each frame is computed, this number reach a maximum when the legs are farthest apart and drop to a minimum when the legs overlap; and the gait period length is the number of frames between two consecutive maxima. To improve the determination of the gait period length, only the lower part of the silhouette (the legs) is used. For each step, the average silhouette is obtained, and for recognition, a similarity function defined as the negative of the median of the Euclidian distance between the average silhouettes of the individual to be classified and a training sequence is used.

An interesting approach is presented by Chai, Wang, Jia, and Zhao (2006) [9], where the silhouettes are extracted using background subtraction, after some morphological methods are applied for noise reduction. The silhouettes are divided into three regions: head region (top 20\%), trunk region (middle 40\%), and legs region (bottom $40 \%$ ). Each silhouette is represented through 4 characteristics, the variance of each body region and the height-width ratio of the body. Using this representation and the gait period length computed as in [8], a similarity function based on the Euclidian distance is defined. Using this function, KNN is used for recognition.

Boulgouris and Chi (2007) [10] proposed a GR method based on body component matching. The silhouettes are extracted and manually divided and labeled in eight regions: head, torso, left arm, right arm, left thigh, right thigh, left leg, right leg. Based on these regions a similarity is defined as a weighted sum of component-tocomponent distances. The different components of the human body were tested separately, showing that they have different discrimination power. Combining the results from all body components gait recognition performance is improved. 
Fazenda, Santos and Correia (2005) [11] proposed a GR method based on dividing each sequence in gait cycle subsequences, then the average silhouette is calculated for each gait cycle. The similarity between two sequences is computed as the median of the Euclidian distances between the subsequence average silhouettes for each gait cycle. Recognition is based on computing the similarity between the sequence to be classified and the training sequences.

Han and Bhanu (2005) [12] proposed a GR method for infrared images based on average silhouettes for each gait cycle. The silhouettes are obtained using background subtraction, and average silhouettes are obtained as in [11]. Then, PCA is applied to reduce feature dimensionality, and after MDA is applied to obtain better class separability. Good recognition accuracy is reported in a database containing only five people.

Tan, Huang, Yu and Tan (2006) [13] present another GR method for infrared images; their method is similar to [12], but they propose removing the bottom of the silhouette and using only the head-torso-thigh region. A similarity function based on the Euclidian distance is used for recognition.

In these works, the GR is done through a similarity function based in a distance function, it has allowed getting good results however, these methods do not offer a unique solution; they provide an ordering on the similarity between the training sequences and the sequence to be classified. Therefore, in this paper we propose two new methods for Gait Recognition based on Silhouette, Contour and Classifier Ensembles giving a unique solution as output.

\section{Proposed Methods}

In this section, two methods for gait recognition using contour and silhouette of a walking person, based on classifier ensembles, are introduced.

Average silhouettes preserve information about the motion and shape of the whole human body during the gait, high intensity pixels represent the most stable part of the body, and low intensity pixels represent the most dynamic parts. In the average silhouettes, most of the motion is captured in the border; therefore most of this motion information could be preserved if only the average contour is considered. Since our method is based on classifier ensembles, using average silhouette and average contour, separately, to train different classifiers will provide diversity, which would help to improve the classification accuracy.

Both proposed methods perform a preprocessing step, where, as in [9], the silhouette is extracted by separating the background from each frame through background subtraction. In order to eliminate noise, the silhouette is binarized and dilation and erosion (morphological operators) are applied. The binary images are standardized and normalized into a uniform size like in [7], i.e. $129 \times 130$ pixels. Due to the average silhouette is a robust representation with respect to segmentation errors and it does not depend on the choice of the starting stance of the gait period [8], we propose to use it, in our methods, as a representation of the gait.

For computing the average silhouette, the gait period length, denoted as $N$, is estimated. Since the width of the silhouette in pixels reaches a maximum when the two 
legs are farthest apart and it drops to a minimum when the legs are overlapped; the gait period length is considered as the number of frames contained between two consecutive maximums. This interval between two consecutive maximums defines one step [9]. Therefore, given a sequence $S$ of binary silhouettes and the gait period length $N$, the sequence is partitioned into sub-sequences $S^{i}$ of gait period length, following [11] for each subsequence (or step) an average silhouette $A S(i)$ is obtained using (1)

$$
A S(i)=\frac{1}{N} \sum_{k=1}^{N} S^{i}(k)
$$

where

$S^{i}(k)$ is the image $k$ in the subsequence $i ; i=1, \ldots,\left\lfloor\frac{T}{N}\right\rfloor$, being $T$ the number of images in the whole gait sequence.

The average silhouettes of all the stored gallery sequences are used as training for an ensemble of classifiers, which will recognize unseen sequences. In our approach, the training sequences and the probe or new sequences to classify must have the same number of average silhouettes. The first ensemble's classifier is trained with the first average silhouette in the sequence; the second classifier is trained with the second

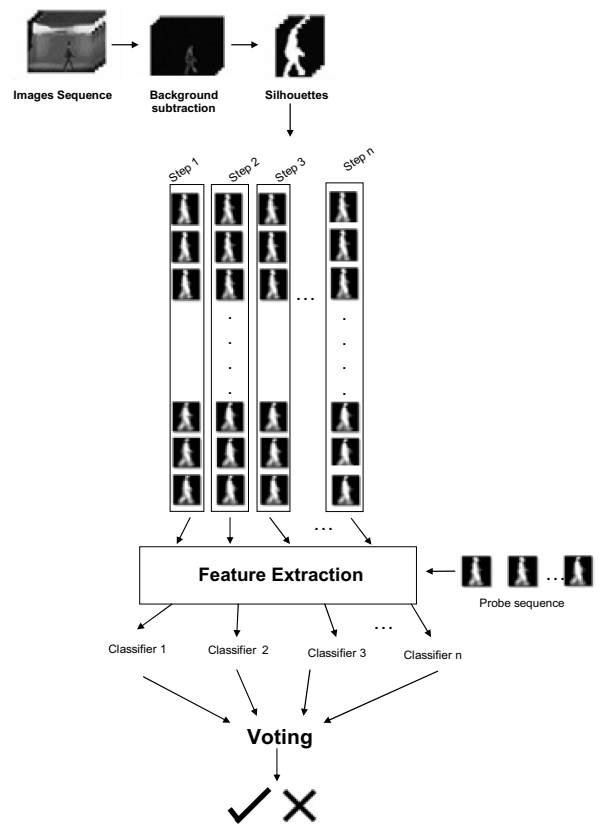

(a)

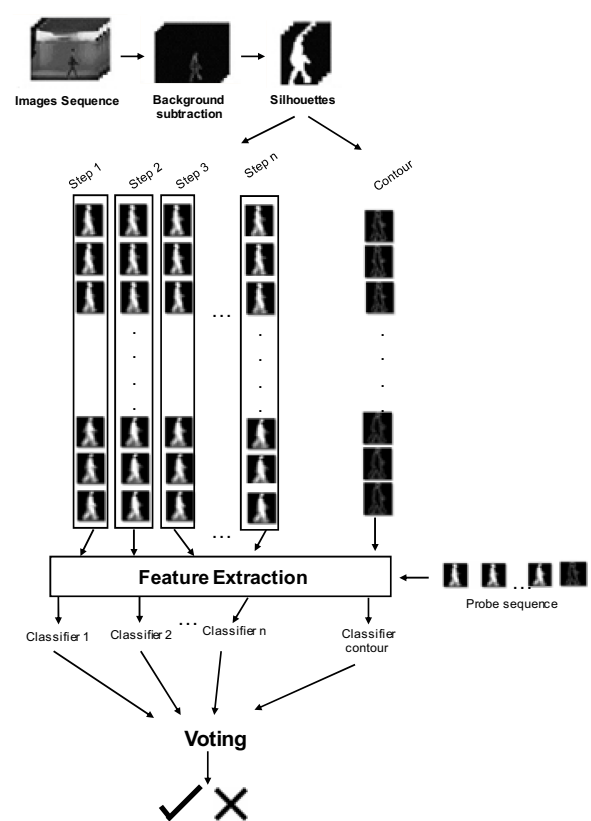

(b)

Fig. 1. (a) Method 1.1, (b) Method 1.2 
average silhouette and so on. The recognizing is performed by majority voting of the results of each classifier.

In our methods, Principal Component Analysis (PCA) and Multiple Discriminant Analysis (MDA) combined, as feature extraction, over the average silhouettes, to get a low dimensional and class separability simultaneously [12], are applied. The whole method can be seen in the figure 1a. A variant of this method considering besides the average silhouettes, the average contour of these silhouettes is shown in the figure $1 \mathrm{~b}$. Adding the average contour implies adding another classifier in the ensemble. In order to classify a new sequence its average silhouettes (and the average contour for the method 2.1) are computed and each one is given to one classifier in the ensemble.

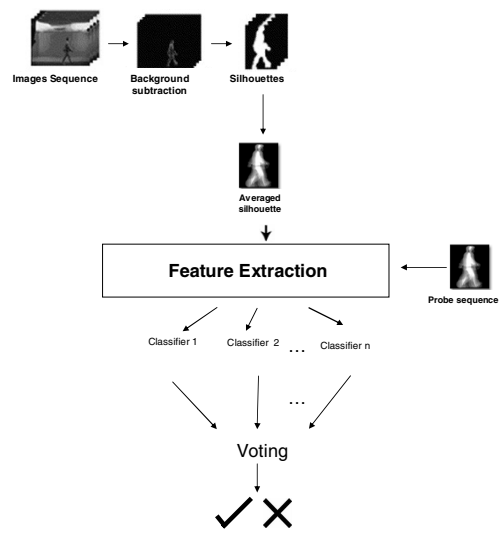

(a)

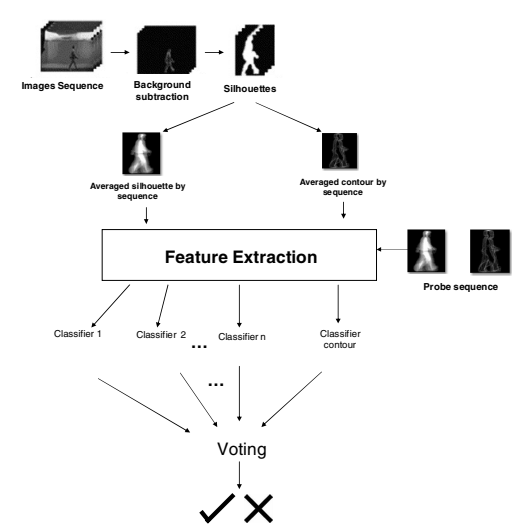

(b)

Fig. 2. (a) Method 2.1, (b) Method 2.2

As a second method, we propose to use the average silhouette but in the whole sequence instead of the average silhouettes per period, see figure $2 \mathrm{a}$. In this case, unlike methods 2.1 and 2.2, the same information (average silhouette) is given to each classifier of the ensemble. In the figure $2 \mathrm{~b}$ a variant of this method considering the average silhouette in the whole sequence and the average contour in the sequence, is shown.

\section{Experimental Results}

For the experimental evaluation of our methods, we used the database CASIA [14]. This database contains human gait sequences in visible (20 persons each one with 4 sequences) and infrared (140 persons each one with 4 sequences). In our experiments, the first three strides were used because all the persons walked at least three strides. After several tests analyzing different classifiers, for method 1.1 KNN, SVM, and KLLDC were chosen for the ensemble. Due to method 1.2 uses the average contour and in order to avoid ties in the voting step, besides classifiers used for average silhouettes in method 1.1, the classifiers KNN and SVM were used for the average contour. In the method 2.1 LDC, SVC, and KLLDC classifiers were chosen in the 
ensemble. While in the method 2.2, besides the classifiers for the silhouette, KLLDC and SVC classifiers were added for the average contour. The use of some of these classifiers has been reported in previous works but not in an ensemble $[11,12,13]$.

The training set for our experiments was built with all the sequences of each person except one which was used as probe sequence. In order to evaluate our methods, this process was repeated $M$ times being $M$ the number of sequences per person and the probe sequence was classified by the ensemble. The average accuracy was computed as the number of correctly classified sequences divided by the total number of sequences per person multiplied by 100 .

Table 1. Results of proposed methods in visible and infrared normal walking sequences

\begin{tabular}{cccc}
\hline Database & Method & Num. of persons & Accuracy \\
\hline & 1.1 & 20 & 91.30 \\
Casia & 1.2 & 20 & 93.50 \\
(Visible) & 2.1 & 20 & 100 \\
& 2.2 & 20 & 100 \\
\hline & 1.1 & 46 & 97.30 \\
& 1.2 & 46 & 99.00 \\
Casia & 2.1 & 46 & 100 \\
(Infrared) & 2.2 & 46 & 100 \\
& 1.1 & 80 & 98.13 \\
& 1.2 & 80 & 99.07 \\
& 2.1 & 80 & 100 \\
& 2.2 & 80 & 100 \\
\hline
\end{tabular}

In table 1, the results obtained with our methods using normal walking sequences (visible and infrared) from CASIA database are shown. The methods 2.1 and 2.2 got the best results having a perfect recognizing in all the experiments. In these experiments always, the method 1.2 was better than the method 1.1 .

Table 2. Comparation of results of our methods against other methods

\begin{tabular}{|c|c|c|c|c|c|c|c|c|c|}
\hline \multirow{2}{*}{ Database } & \multirow{2}{*}{$\begin{array}{l}\text { Num. } \\
\text { of } \\
\text { person }\end{array}$} & \multirow{2}{*}{$\begin{array}{l}\text { Recognizing } \\
\text { by }\end{array}$} & \multicolumn{4}{|c|}{ Method } & \multirow{2}{*}{ [11] } & \multirow{2}{*}{ [13] } & \multirow{2}{*}{ [12] } \\
\hline & & & 1.1 & 1.2 & 2.1 & 2.2 & & & \\
\hline \multirow{2}{*}{$\begin{array}{c}\text { Casia } \\
\text { (visible) }\end{array}$} & \multirow{2}{*}{20} & Sequence & 91.3 & 93.5 & 100 & 100 & 83.8 & 88.8 & 43.8 \\
\hline & & Person & 95.0 & 95.0 & 100 & 100 & 90.0 & 85.0 & 30.0 \\
\hline \multirow{2}{*}{$\begin{array}{c}\text { Casia } \\
\text { (Infrared) }\end{array}$} & \multirow{2}{*}{46} & Sequence & 97.3 & 99.0 & 100 & 100 & 99.0 & 96.0 & 96.2 \\
\hline & & Person & 100 & 100 & 100 & 100 & 100 & 100 & 97.9 \\
\hline
\end{tabular}

In table 2, a comparison of our methods against the methods reported in $[11,12,13]$ are shown. It is important to point out that all the methods (proposed and reported) were implemented and tested on the same computer. For all the methods, the same DB having figures in the same format and size as the same evaluating method were used. In this experiment, in addition to the accuracy, the recognizing in terms of the persons correctly classified was computed, in this case a person was considered as recognized if more than the half of sequences for this person was correctly classified, in our experiments if three sequences of its four sequences were correctly classified. 
Table 3. Results training with normal walking sequences and classifying with quick and slow walking sequences

\begin{tabular}{|c|c|c|c|c|c|c|c|c|c|}
\hline \multirow{2}{*}{ Database } & \multirow{2}{*}{$\begin{array}{l}\text { Num } \\
\text { persons }\end{array}$} & \multirow{2}{*}{ Experiment } & \multicolumn{4}{|c|}{ Method } & \multirow[t]{2}{*}{ [11] } & \multirow[t]{2}{*}{ [13] } & \multirow[t]{2}{*}[12]{} \\
\hline & & & 1.1 & 1.2 & 2.1 & 2.2 & & & \\
\hline \multirow{2}{*}{$\begin{array}{c}\text { Casia } \\
\text { (Infrared) }\end{array}$} & \multirow{2}{*}{46} & $\begin{array}{l}\text { Training: } \\
\text { normal } \\
\text { Test: slow }\end{array}$ & 99.0 & 99.0 & 97.9 & 93.5 & 83.7 & 74.0 & 54.4 \\
\hline & & $\begin{array}{l}\text { Training: } \\
\text { normal } \\
\text { Test: quick }\end{array}$ & 93.5 & 93.5 & 93.5 & 84.9 & 91.4 & 90.0 & 70.7 \\
\hline
\end{tabular}

As it is possible to see, from table 2, the best results again were obtained by the methods 2.1 and 2.2. While the methods 1.1. and 1.2 outperformed the methods proposed in $[11,12,13]$.

In the last experiment, the classifiers were trained with normal walking sequences and then slow and quick walking sequences were classified. The best results of accuracy were obtained by methods 1.1 and 1.2 , see table 3 . In this case, the methods 2.1 and 2.2 outperformed the methods proposed in $[11,12,13]$.

\section{Conclusions}

In this paper, two new methods for Gait Recognition based on silhouette and contour, using a classifier ensemble were introduced. Based in our experiments we can conclude that using contour, silhouette and combining different classifiers in an ensemble it was possible to get better recognizing accuracy in visible and infrared sequences than other recent similar methods. The best results for normal walking were obtained by our methods 2.1 and 2.2 .

In our last experiment, when the ensemble was trained with infrared normal walking sequences and slow and quick walking sequences were classified, the best result was obtained by the method 1.1 followed by method 1.2.

As future work, we are going to test other feature extraction methods and the use of other classifiers in the ensemble in order to try improving the accuracy for sequences in the visible. In addition, we will test our methods with sequences taken in different camera angle and in sequences of different gait types as slow walking, quick walking, jog and run.

\section{References}

[1] Jain, A.K.: Biometric Recognition: Overview and Recent Advances. In: Rueda, L., Mery, D., Kittler, J. (eds.) CIARP 2007. LNCS, vol. 4756, pp. 13-19. Springer, Heidelberg (2007)

[2] Nixon, M.S., Carter, J.N.: Automatic Recognition by Gait. Proceedings of the IEEE 94(11), 2013-2024 (2006)

[3] Nixon, M.S., Carter, J.N., Nash, J.M., Huang, P.S., Cunado, D., Stevenage, S.V.: Automatic gait recognition. In: IEE Colloquium Motion Analysis and Tracking, pp. 3/1-3/6 (1999) 
[4] Murray, M.: Gait as a total pattern of movement. American J. of Physical Medicine 46(1), 290-332 (1967)

[5] Dawson, M.R.: Gait recognition, Final Report. Department of Computing Imperial College of Science, Technology \& Medicine, London (2002)

[6] Lam, T.H.W., Lee, R.S.T., Zhang, D.: Human gait recognition by the fusion of motion and static spatio-temporal templates. Pattern Recognition 40(9), 2563-2573 (2007)

[7] Wang, L., Tan, T., Ning, H., Hu, W.: Silhoutte analysis based gait recognition for human identification. IEEE trans Pattern Analysis and Machine Intelligence 25(12), 1505-1518 (2003)

[8] Liu, Z., Sarkar, S.: Simplest representation yet for gait recognition: averaged silhouette. In: IEEE International Conference on Pattern Recognition, pp. 211-214. IEEE Press, Cambridge (2004)

[9] Chai, Y., Wang, Q., Jia, J., Zhao, R.: A Novel Human Gait Recognition Method by Segmenting and Extracting the Region Variance Feature. In: 18th International Conference on Pattern Recognition, Hong Kong, vol. 4 (2006)

[10] Boulgouris, N.V., Chi, Z.X.: Human gait recognition based on matching of body components. Pattern Recognition 40(6), 1763-1770 (2007)

[11] Fazenda, J., Santos, D., Correia, P.: Using Gait to Recognize People. In: Computer as a Tool, EUROCON 2005, vol. 1, pp. 155-158 (2005)

[12] Han, J., Bhanu, B.: Human Activity Recognition in Thermal Infrared Imagery. In: IEEE Computer Society Conference on Computer Vision and Pattern Recognition, San Diego (2005)

[13] Tan, D., Huang, K., Yu, S., Tan, T.: Efficient Night Gait Recognition Based on Template Matching. In: 18'th International Conference on Pattern Recognition (ICPR 2006), Hong Kong (2006)

[14] CASIA Gait Database, http://www.sinobiometrics.com 